\title{
Artificial Immune System Based Local Search for Solving Multi-Objective Design Problems
}

\author{
Adel M. El-Refaey \\ Basic and Applied Science Department, College of Engineering and Technology, Arab Academy for Science, Technology and Maritime \\ Transport, Cairo, Egypt
}

Email address:

adel_elrefaey@aast.edu

To cite this article:

Adel M. El-Refaey. Artificial Immune System Based Local Search for Solving Multi-Objective Design Problems. American Journal of Neural Networks and Applications. Vol. 3, No. 3, 2017, pp. 29-35. doi: 10.11648/j.ajnna.20170303.11

Received: October 24, 2017; Accepted: November 20, 2017; Published: December 14, 2017

\begin{abstract}
In this paper, an artificial intelligent approach based on the clonal selection principle of Artificial Immune System (AIS) and local search (LS) is propose to solve Multiobjective engineering design problems. This paper presents an optimal design of a linear synchronous motor (LSM) considering two objective functions namely, maximum force and minimum saturation and then design of air-cored solenoid with maximum inductance and minimum volume as the objective functions. The proposed approach uses Local search, dominance principle and feasibility to identify solutions that deserve to be cloned.
\end{abstract}

Keywords: Artificial Immune System, Local Search, Multiobjective Programming, Clonal Selection, Design Optimization

\section{Introduction}

Computing and engineering have been enriched by the introduction of the biological ideas to help developing solutions for various problems. [7]

Artificial Immune Systems (AIS) are computational paradigms that belong to the computational intelligence family and are inspired by the biological immune system. [5]

Clonal selection theory was proposed by Burnet (1959). The theory is used to explain basic response of adaptive immune system to antigenic stimulus. It establishes the idea that only those cells capable of recognizing an antigen will proliferate while other cells are selected against. Clonal selection operates on both B and T cells. B cells, when their antibodies bind with an antigen, are activated and differentiated into plasma or memory cells. Prior to this process, clones of B cells are produced and undergo somatic hyper mutation. As a result, diversity is introduced into the $\mathrm{B}$ cell population. Plasma cells produce antigen-specific antibodies that are work against antigen. Memory cells remain with the host and promote a rapid secondary response. The number of clones for the pool of best antibodies depends on the antibody-antibody affinity. These best antibodies are selected for a uniform mutation, with mutation probability proportional to antibody-antigen affinity, according to the ranking scheme, while the remaining population undergoes a non-uniform mutation. Again, the ranking scheme is used as criterion to reduce the population to its original cardinality. [11, 13]

Local search techniques have been very popular as heuristics for hard combinatorial optimization problems. The basic idea is to start from an initial solution and to search for successive improvements by examining neighboring solutions. The local search used in this paper is based on a dynamic version of pattern search technique. Pattern search technique is a popular paradigm in Direct Search (DS) methods [4].

The growth in demand for linear motors is principally driven by the replacement of traditional mechanical (ball screws, gear trains, cams), hydraulic, or pneumatic linear motion systems in manufacturing processes, machining, material handling, and positioning with direct electromechanical drives. The linear synchronous motor (LSM) operates on the same working principle as that of a permanent magnet rotary D. C. motor [2, 3]. As in a rotary motor there are two parts in a LSM, one is the set of permanent magnets and the other is the armature that has conductors carrying current. The permanent magnets produce a magnetic flux perpendicular to the direction of motion. The flow of current is in the direction perpendicular to both the direction of the motion and the direction of the magnetic flux. [1]

This paper intends to present an optimal design of a LSM to replace a hydraulic actuator and design air-cored solenoid 
using hybrid AIS approach. This methodology combined AIS with Local Search, AIS find initial Pareto front then Local search improve this initial Pareto after that applying mutation process. Results show that the combination between AIS and LS improve the solution quality of Multiobjective design optimizations and explore large area in objectives space.

The remainder of the paper is organized as follows. In Section 2 describe some preliminaries on Multiobjective optimization problem (MOP). In Section 3 review the artificial immune system. In Section 4 explain a local search technique, in section 5 present the proposed approach. Experimental results are given and discussed in Section 6. Section 7 indicates conclusion.

\section{Preliminaries}

A general Multiobjective optimization problem is expressed as follows: [16]

MOP:

$$
\begin{gathered}
\text { Min } F(x)=\left(f_{1}(x), f_{2}(x), \ldots, f_{k}(x)\right)^{t} \\
\text { s.t. } \quad x \in S
\end{gathered}
$$

Where $(k \geq 2)$ objective functions $f_{i}: R^{n} \rightarrow R$, the decision (variable) vector $x=\left(x_{1}, x_{2}, \ldots, x_{n}\right)^{t}$ belongs to the (nonempty) feasible region (set) $\mathrm{S}$, which is a subset of the decision variable space $R^{n}$. [10]

Definition 1 (Pareto optimal solution): $x^{*}$ is said to be a Pareto optimal solution of MOP If there exists no other feasible $x$ (i.e., $x \in S)$ such that, $f_{j}(x) \leq f_{j}\left(x^{*}\right)$ for all $j=1,2, \ldots, k$ and $f_{j}(x)<f_{j}\left(x^{*}\right)$ for at least one objective function $f_{j}$. [10]

The terms "dominance" and "Pareto optimality" can be mathematically defined for a general problem of simultaneously minimizing a $k$-components vector function $F=\left(f_{1}(x), f_{2}(x), \ldots, f_{k}(x)\right)$ of an $n$ dimensional decision variable vector $x=\left(x_{1}, x_{2}, \ldots, x_{n}\right)$ from some universe $S$ (figure $1)$.

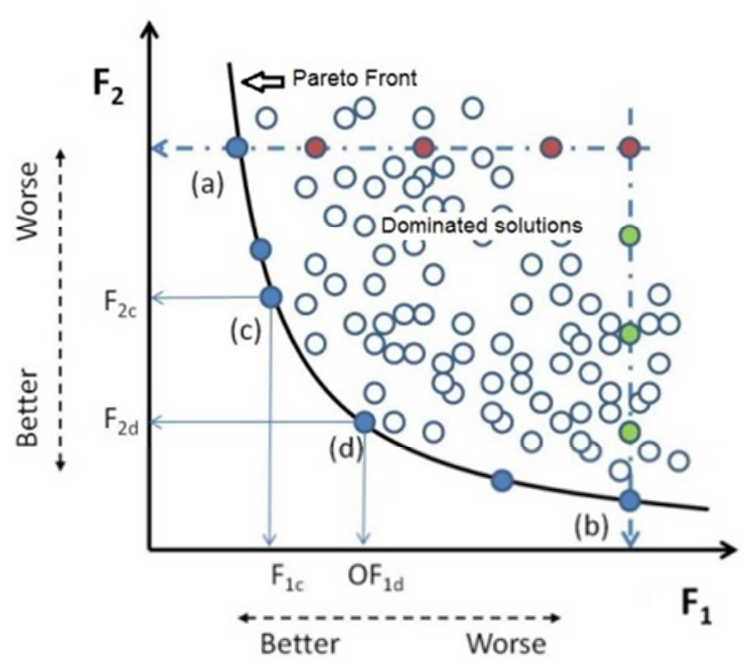

Figure 1. The concept of Pareto optimality.

\section{Artificial Immune Systems}

The main goal of the immune system is to protect the human body from the attack of foreign (harmful) organisms. The immune system is capable of distinguishing between the normal components of organism and the foreign material that can cause us harm (e.g. bacteria). These foreign organisms are called antigens (Ag's). The molecules called antibodies (Ab's) play the main role on the immune system response. The immune response is specific to a certain foreign organism (antigen). When an antigen is detected, those antibodies that best recognize an antigen will proliferate by cloning. This process is called clonal selection principle, the new cloned cells undergo high rate of mutation. [6].

In Natural Immune System (NIS) research, four models of the NIS can be found:

1. The classical view of the immune system is that the immune system distinguishes between self and nonself, using lymphocytes produced in the lymphoid organs. These lymphocytes "learn" to bind to antigen. [8]

2. Clonal selection theory, where an active B-Cell produces antibodies through a cloning process. The produced clones are also mutated.

3. Danger theory, where the immune system has the ability to distinguish between dangerous and nondangerous antigen.

4. Network theory, where it is assumed that B-Cells form a network. When a B-Cell responds to an antigen, that B-Cell becomes activated and stimulates all other BCells to which it is connected in the network. [12]

5. Clonal Selection Theory

One example of a cellular evolution is the development of the B cell (and T cell) immune repertoire. B and T cells are cells of the adaptive immune response. In contrast to the innate immune response, which is always ready to respond to whatever intruder, the adaptive immune response matures throughout life, is antigen (Ag) specific and long-living. The specificity of $\mathrm{B}$ cells lies in the variable region of their antibodies, each B cell produces antibodies (Ab's) with one particular specificity. [13]

$\mathrm{Ab}$ 's are molecules attached primarily to the surface of $\mathrm{B}$ cells whose aim is to recognize and bind to Ag's. Each B cell secretes a single type of $\mathrm{Ab}$, which is relatively specific for the Ag. By binding to these Ab's and with a second signal from accessory cells, such as the T-helper cell, the Ag stimulates the $\mathrm{B}$ cell to proliferate (divide) and mature into terminal (nondividing) Ab secreting cells, called plasma cells. The process of cell division (mitosis) generates a clone, i.e., a cell or set of cells that are the progenies of a single cell. $\mathrm{B}$ cells, in addition to proliferating and differentiating into plasma cells, can differentiate into long-lived B memory cells. Memory cells circulate through the blood, lymph, and tissues and, when exposed to a second antigenic stimulus, commence to differentiate into plasma cells capable of producing high-affinity Ab's, preselected for the specific Ag that had stimulated the primary response. Figure. 2 depicts 
the clonal selection principle. [12]

The main features of the clonal selection theory $[2,10]$ that will be explored in this paper are:

1) Proliferation and differentiation on stimulation of cells with Ag's.

2) Generation of new random genetic changes, expressed subsequently as diverse $\mathrm{Ab}$ patterns, by a form of accelerated somatic mutation (a process called affinity maturation).

3) Estimation of newly differentiated lymphocytes carrying low-affinity antigenic receptors.

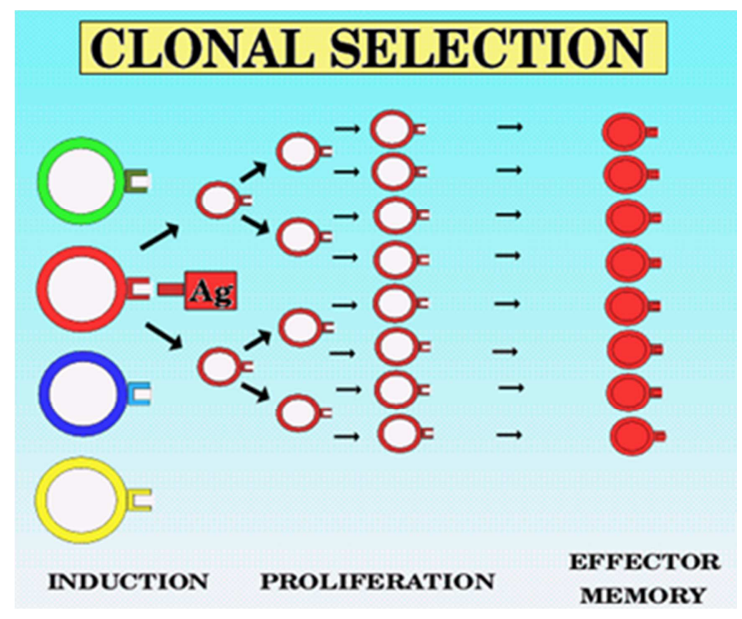

Figure 2. Clonal selection principle.

\section{Local Search}

The local search phase is implemented as a dynamic version of pattern search technique. Pattern search technique is a popular paradigm in Direct Search (DS) methods. DS methods are evolutionary algorithms used to solve constrained optimization problems. [4]

This study examines the importance of a dynamic version of pattern search technique to improve the solution quality of MOPs. The search procedure looks for the best solution "near" in the neighborhood of the current solution by repeatedly making small changes to a starting solution. The local search is started by loading the Pareto solutions for a given MOPs. At iteration $t$, an iterate $x_{t} \in$ Pareto is obtaoned, where the changes on the values for each dimension $(i=1,2, \cdots, n)$ can be implemented as

$$
\Delta t=R\left(1-r^{(1-t / T)}\right)
$$

where $\mathrm{r}$ is the random number in the range $[0,1]$; $\mathrm{T}$ is the maximum number of iterations; $\mathrm{R}$ is the search radius. Let $e_{i},(i=1,2, \cdots, n)$, denote the standard unit basis vectors. Successively look at the points $x_{+}=x_{t} \pm \Delta t \cdot e_{i},(i=1,2, \cdots, n)$, until finding $x_{+}$for which $f\left(x_{+}\right)$dominates $f\left(x_{t}\right)$ for at least one objective, If the dominance not satisfied then $x_{+}=x_{t}$. Then update the Pareto solutions by non dominated ones and the dominated ones are removed. This situation is represented in Figure 3. [1]

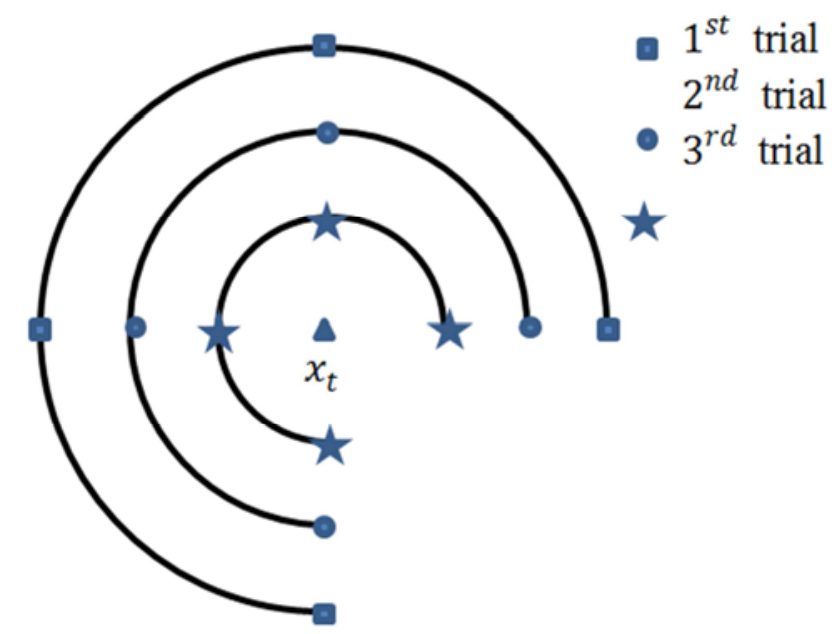

Figure 3. Mechanism of dynamic pattern search in $R^{2}$.

\section{The Proposed Approach}

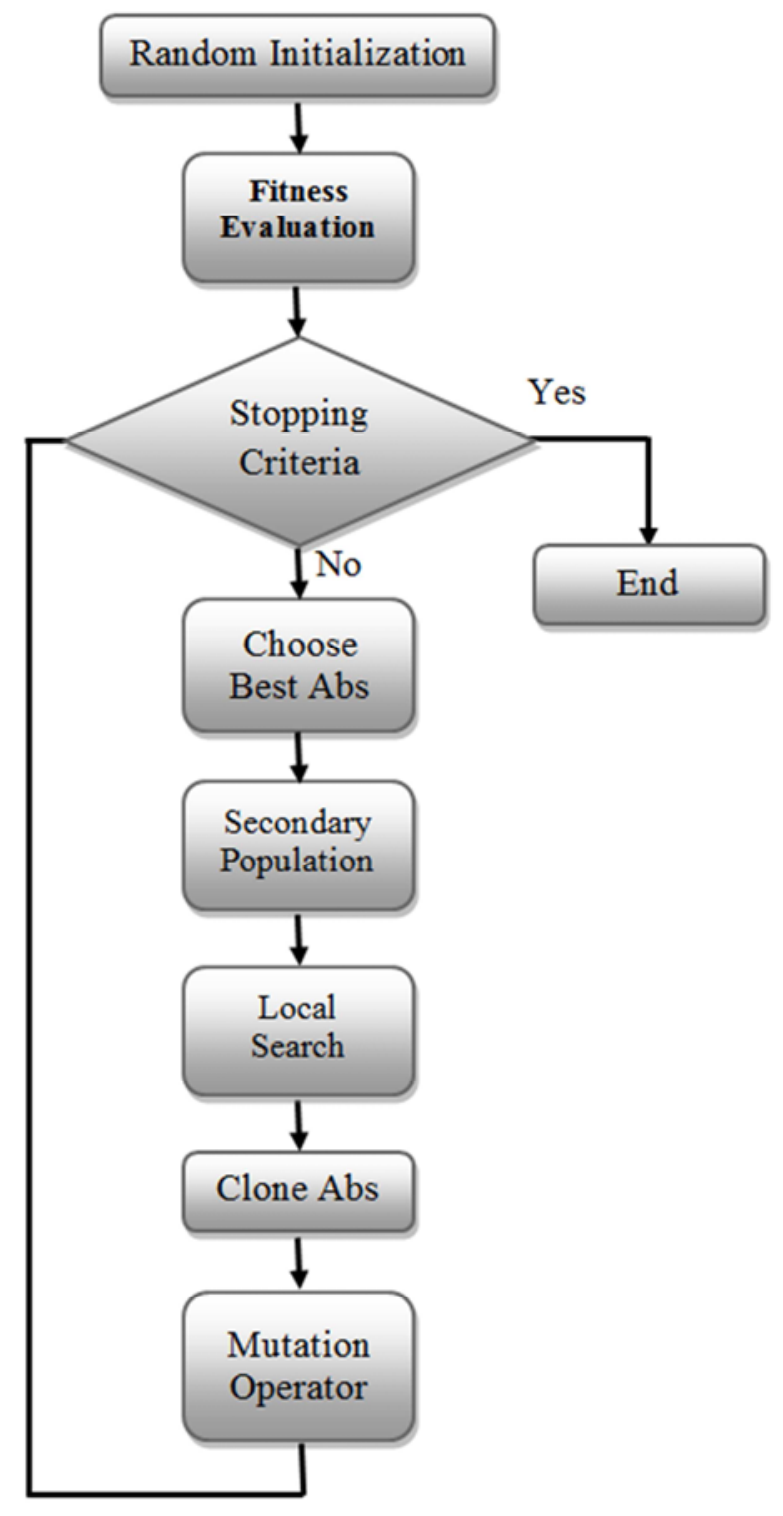

Figure 4. The proposed algorithm. 
The algorithm run with random input to AIS which has taken ideas from the clonal selection principle, [17] modeling the fact that only the highest affinity antibodies will go through local search algorithm then proliferate. Antibodies, in this case, are represented by decimal value which represent the value of decision variables of the problem to be solved. However, not using a population of antigens, but only Pareto dominance and feasibility to identify solutions that deserve to be cloned. Additionally, theproposed approach uses mutation [8] (uniform mutation is applied to the clones and non-uniform mutation is applied to the "not so good" antibodies). Also using a secondary (or external) population that stores the nondominated solutions found along the search process. Such secondary population is the elitist mechanism most commonly adopted in multiobjective optimization, and it allows us to move towards the Pareto front [7].

The algorithm

The proposed algorithm for solving multiobjective design problems using Multiobjective Immune System Algorithm (MISA) with Local search is as follow:

[Step 1] Random Initialization

[Step 2] Sorting population according to dominance

[Step 3] Choose the "best" antibodies to be cloned (nondominated Antibodies)

[Step 4] Apply local search for "best" antibodies to find the "best of best" Antibodies

[Step 5] Cloning "the best of best" antibodies

[Step 6] Appling a uniform mutation to the clones

[Step 8] Repeat this process from step 2 till reach the desired number of antibodies

\section{Numerical Results}

In order to validate the proposed approach, it used to solve two engineering design problems.

\subsection{Shape Design of a Linear Synchronous Motor}

A linear motor is an electric motor that has its stator and rotor "unrolled" so that instead of producing a torque (rotation) it produces a linear force along its length. To permit more flexibility of operation and allow short headways for high-capacity operation, a design has been proposed with very short stator sections. With appropriate design, the operation control system (signaling system) can be integrated with the power feeding system. [14]

The task is to design a direct electrical drive actuator as an alternative to hydraulic cylinder drive. The force can be calculated using the so-called Bli law, which says that the force is the product of the flux density, the length of the conductors and the current through the conductors. To increase the force one needs to either increase the flux density, the length of the conductors or the total current. At the same time one has to consider the inherent as well as external limitations that appear in the form of constraints such as: $[1,15]$

a) Heat Constraint: The preliminary calculations for the
LSM show that approximately $4000 \mathrm{~W}$ of heat can be dissipated out of the motor with the proposed arrangement of coils. Considering $4000 \mathrm{~W}$ as the upper limit on the heat dissipation rate.

b) Radius Constraint: Usually the geometry and the total volume available restrict the size of the LSM. This sets a limit of the total radius of the LSM consists of the magnets, the air gap, back iron in the circuit and the conductor slots.

c) Saturation Constraint: Once the iron is saturated, increasing the magnetic field strength is not useful as it will not increase the amount flux and hence the force will not increase. This sets a limit on how much the magnetic field strength can be usefully increased. The tooth in the stator has the least cross-sectional area and will saturate first.

d) Demagnetization Constraint: Very high values of the armature current will produce a very large opposing magnetic field which may demagnetize the magnets permanently thus altering the motor performance. This is one more limit on the armature current.

e) Minimum Force Requirement: it is required to produce a force greater than that available from any commercially available motor, a minimum force constraint on this value can be based.

Mathematical Problem Statement [1]

Consider the LSM to be a three phase motor with two phases conducting at any point in time. The conductors are assumed to be copper conductors and the permanent magnets to be high density Neodium Iron Boron magnets. The fill factor, $k_{\text {fill }}$, the fraction of slot volume occupied by conductors, is assumed to be equal to 0.6. Formal derivations of the mathematical expressions for the force generated in a LSM and the constraints are carried out in [1]. The analysis is simplified with the following assumptions.

Two variables, current, $i$, through each conductor and the number of conductors, $n s$, in each slot, appear in three expressions, namely the force expression, the heat constraint expression and the demagnetization constraint expression. In all three expressions $i$ and $n s$ appear together as $\left(i^{*} n s\right)$. Replace these two variables by a single variable named slot current, ins, to simplify calculations.

The air gap flux density is monotonically decreasing with the air gap length. There is no advantage of increasing the air gap length and hence one would like to keep it as small as possible. However, it is always difficult to maintain a very small air gap especially in case of the linear motors. Assume the air gap length to be equal to $1 \mathrm{~mm}$ which is the lowest allowed in light of manufacturing considerations.

The back iron on the stator side and the mover side close the flux loop as shown in Figure 5. The back iron carries high flux and is susceptible to saturation. Choose the back iron thickness such that the back iron cross-sectional area is as great as the tooth cross sectional area. Thus avoiding the tooth saturation, ensured by the saturation constraint, will surely eliminate the possibility of the back iron saturation. 


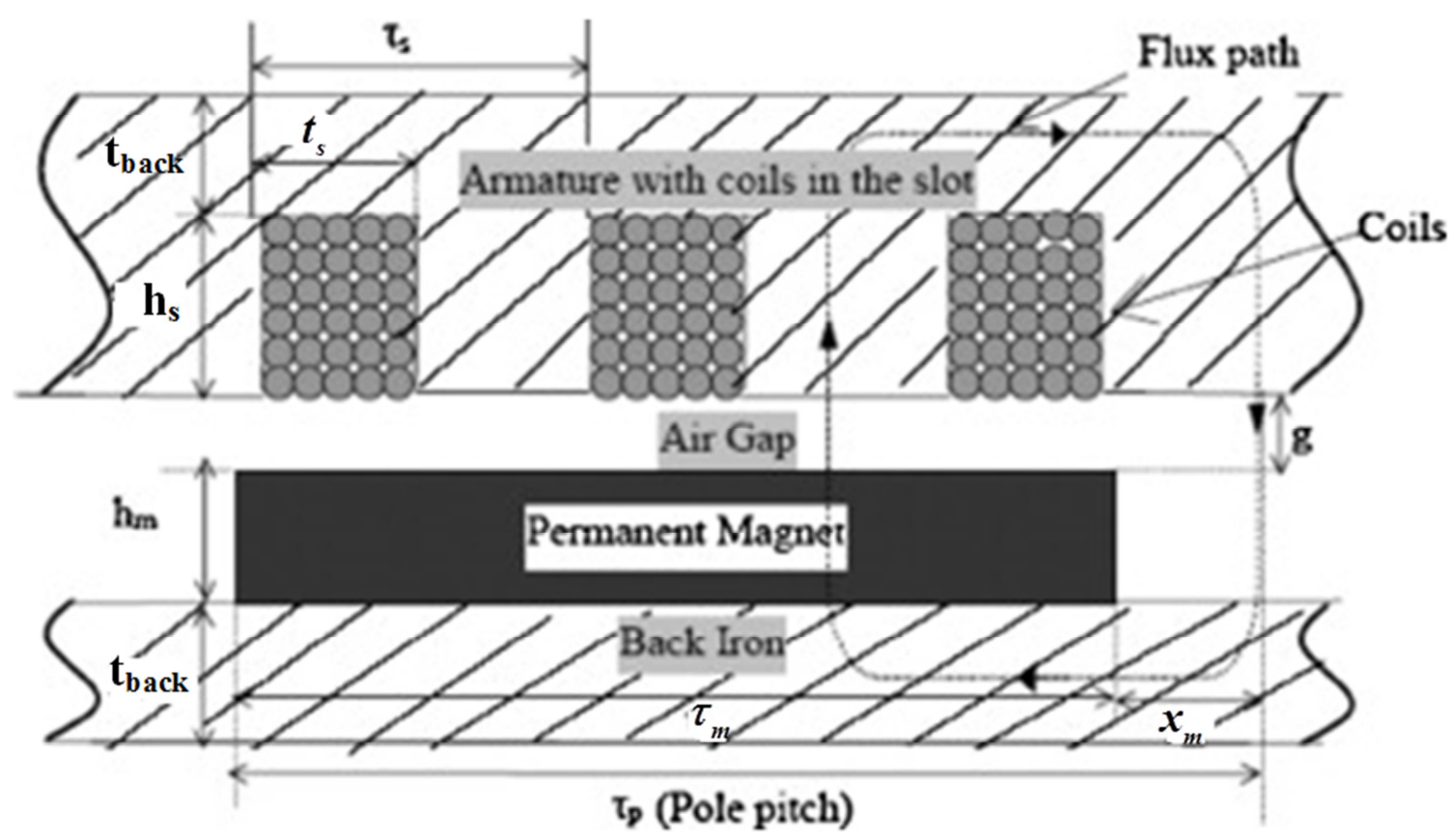

Figure 5. Cross-section of approximately a one pole pitch long section of a Tubular LSM, not to scale.

The total length of the LSM consists of many magnetic poles each of length $\tau_{p}$, the pole pitch, which is a design parameter. The pole pitch, $\tau_{p}$, and the number of poles, $\mathrm{p}$, are related as: (Total Length $=\tau_{p} * \mathrm{p}$ ). In a three phase motor each pole pitch length consists of three slots and three teeth as shown in Figure. 5. The force generated by the LSM, given by the Bli law, has no explicit dependency on $\tau_{p}$ and only varies to the extent that $\mathrm{B}, 1$ or $\mathrm{i}$ vary with $\tau_{p}$. Many small magnets or fewer long magnets yield the same flux density, B, and also many small width slots or few wider slots permit the same current. However, change in $\tau_{p}$ affects the force indirectly, such as an increase in $\tau_{p}$ results in an increase in the back iron thickness to avoid saturation. An increase in the back iron thickness, for a fixed radius, means reduction in slot height resulting in less current for fixed heat or it means thinned magnets resulting in smaller B or both. Thus an increase in $\tau_{p}$ results in a decrease in the force and hence one would like to keep $\tau_{p}$ as small as possible.

The advantage of reducing $\tau_{p}$ continues until $\tau_{p}$ is so small that the magnets (whose length must be $2 / 3$ of $\tau_{p}$ to couple the 2 windings which are "on") become so close to each other that flux leaks from magnet to magnet. This does not happen until magnet spacing is on the order of twice the gap thickness which corresponds to a $\tau_{p}$ well below the $25 \mathrm{~mm}$ allowed in light of manufacturing considerations. Since the number of poles has to be an even integer and since Total Length $=1.09 \mathrm{~m}$, choose $\mathrm{p}=40$ and $\tau_{p}=27 \mathrm{~mm}$. [1]

The relevant design variables are the current in each slot, ins, the dimensions of the slot, $h_{s}$ and $t_{s}$, and the height of the magnet, $h_{m}$. In the current design there are inherent limits on the values of the variables. For example the height of the magnet, $h_{m}$, and the height of the slot, $h_{s}$, are both limited by the radius of the motor. The slot thickness $t_{s}$ can be as large as $1 / 3$ rd of the pole pitch. All lengths are in meters (m), the slot current ins is expressed in Amperes (A) and the flux density is expressed in Tesla $(\mathrm{T})$. The units of the various constants in the equations are not shown explicitly.

$\operatorname{Max} f_{1}=\frac{653.541 * i n s * h_{m}\left(0.02825+h_{m}+\left(h_{s} / 2\right)\right)}{h_{m}+0.0017}$

$\operatorname{Min} f_{2}=\frac{1.3 h_{m}}{h_{m}+0.0017}+165.13 t_{s}$

s. t $g_{1}=\frac{1.9 * 10^{-5} * i n s^{2} * h_{m}\left(0.02825+h_{m}+\left(h_{s} / 2\right)\right)}{h_{s} * t_{s}}-4000 \leq 0$

$g_{2}=h_{m}+h_{s}-3 * t_{s}-0.0266 \leq 0$

$g_{3}=\frac{6.28 * 10^{-7} * \operatorname{ins}\left(h_{m}+0.00105\right)}{h_{m}\left(h_{m}+0.001\right)}-1.17 \leq 0$

$g_{4}=15000-f_{1} \leq 0$

The variables limits are $0 \leq i n s<\infty \mathrm{A}, 0 \leq t_{s} \leq 0.009 m$, $0 \leq h_{s} \leq 0.08 m$ and $0 \leq h_{m} \leq 0.08 m$. Pareto Front using proposed approach is shown in figure 6 a. 


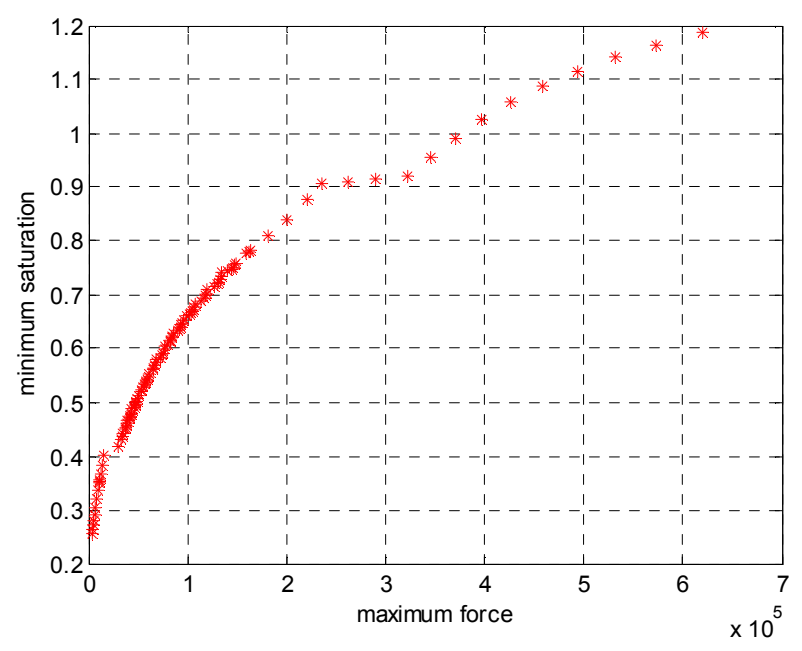

Figure 6a. Pareto Front obtained by using proposed approach.

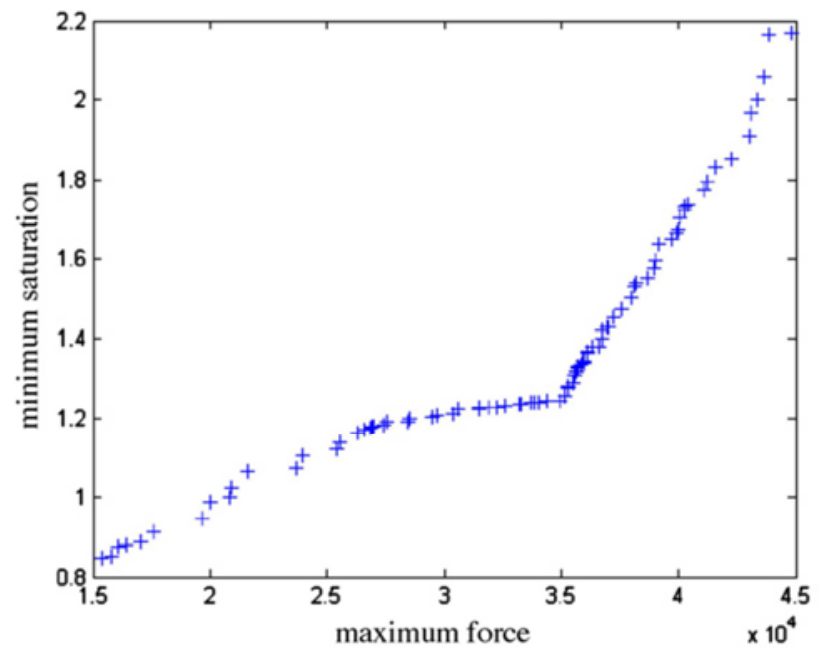

Figure 6b. Pareto front by combining MACO and Local search [1].

\subsection{Shape Design of an Air-Cored Solenoid}

A multiobjective shape optimization problem of a coreless solenoid of rectangular cross-section $b \times c$ and mean radius $a$ is tackled (figure 7).

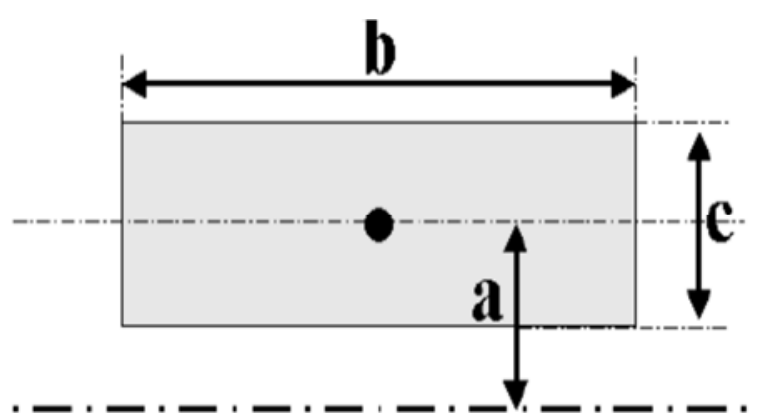

Figure 7. Cross section of the solenoid and design variables.

If current is supposed to be uniformly distributed over the cross-section,

given the geometry of the solenoid and the number $\mathrm{N}$ of turns, the inductance $\mathrm{L}[\mu \mathrm{H}]$ can be approximated by the following formula:

$$
L=\frac{31.49\left(a^{2} N^{2} / b\right)}{9+6(a / b)+10(c / b)}
$$

The multiobjective design problem can be cast in these terms: maximize inductance $\mathrm{L}(\mathrm{a}, \mathrm{b}, \mathrm{c})$ and minimize volume $\mathrm{V}(\mathrm{a}, \mathrm{b}, \mathrm{c})$ for given length $k_{1}=10 \mathrm{~m}$ and cross section $k_{2}=10^{-6} \mathrm{~m}^{2}$ of the current carrying wire. Due to constraints, two variables only, e.g. a and b, can be considered; finally functions $\mathrm{L}$ and $\mathrm{V}$ become [14]

$$
\begin{aligned}
& \operatorname{Max} f_{1}=\frac{31.49\left(k_{1}^{2} / 4\right)}{9+6(a / b)+5\left(k_{1} k_{2} / \pi a b^{2}\right)} \\
& \text { Min } f_{2}=\frac{\pi a^{2} b}{4}+\frac{k_{1}^{2} k_{2}^{2}}{4 \pi a^{2} b}+\frac{k_{1} k_{2}}{2} \\
& \text { s.t } g_{1}=\sqrt{\frac{k_{1} k_{2}}{4 \pi b}}-a<0
\end{aligned}
$$

The variables limits are $0 \leq a \leq 0.1,0 \leq b \leq 0.3$,

Pareto Front using proposed approach is shown in figure $8 \mathrm{a}$.

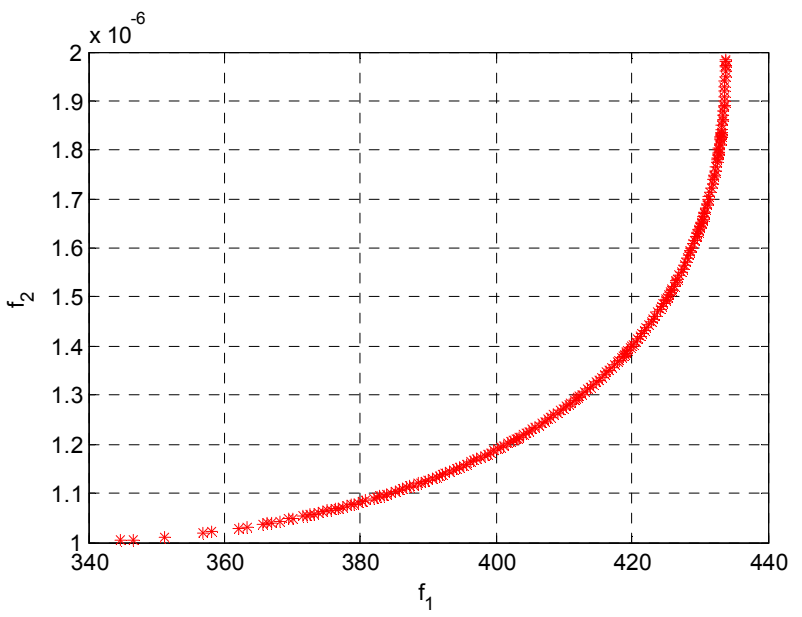

Figure 8a. Pareto Front obtained by using proposed approach.

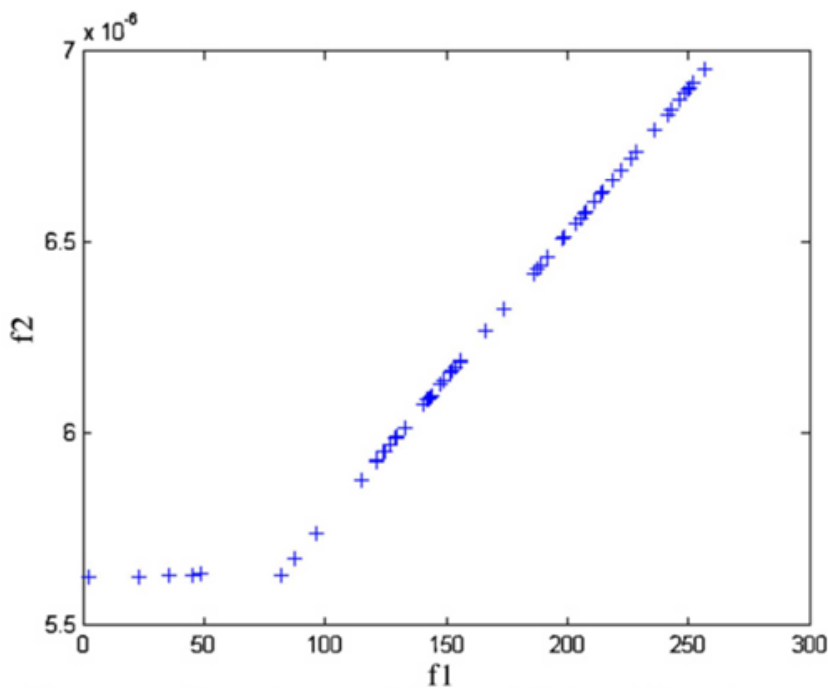

Figure 8b. Pareto front by combining MACO and Local search [1]. 


\section{Conclusion}

A hybrid multiobjective optimization algorithm based on the clonal selection principle and local search have been presented. The approach is able to produce results similar or better than those generated by other evolutionary algorithms and the Pareto optimal Solution more accurate and faster. The proposed approach uses an affinity measure to control the amount of mutation to be applied to the antibodies. Affinity in this case, is defined in terms of nondominance and feasibility.

The proposed approach also uses a very simple mechanism to deal with constrained test functions, and results indicate that such mechanism, despite its simplicity, is effective in practice.

In the two design problems the proposed approach explore large objective space that other evolutionary algorithms.

\section{References}

[1] A. A. Mousa et al, (2011), "A hybrid ant colony optimization approach based local search scheme for multiobjective design optimizations", Electric Power Systems Research, vol.81, pp. 1014-1023.

[2] A. D. Deshpande, J. R. Rinderle, (2001), "Linear Electric Drive for UMM", Technical Report, Department of Mechanical and Industrial Engineering, University of Massachusetts, Amherst.

[3] A. D. Deshpande, (2002), "A Study of Methods to Identify Constraint Dominance In Engineering Design Problems", $M$. S. Thesis, Mechanical and Industrial Engineering Department, University of Massachusetts, Amhers, California Linear Drives, Inc.

[4] A. M. Arasomwan and A. O. Adewumi, (2014), "Improved Particle Swarm Optimization with a Collective Local Unimodal Search for Continuous Optimization Problems," The Scientific World Journal, Volume 2014, Article ID 798129, 23 pages.

[5] C. A. Coello Coello, (2002), "Theoretical and numerical constraint handling techniques used with evolutionary algorithms: A survey of the state of the art," Computer Methods in Applied Mechanics and Engineering, vol. 191, no. 11/12, pp. 1245-1287.

[6] C. A. Coello Coello and N. Cruz Cort'es, (2002) "An approach to solve multiobjective optimization problems based on an artificial immune system", in First International Conference on Artificial Immune Systems (ICARIS'2002), J.
Timmis and P. J. Bentley (Eds.), University of Kent at Canterbury: UK, Sept. 2002, pp. 212-221. ISBN 1-902671$32-5$.

[7] C. A. Coello Coello, D. A. Van Veldhuizen, and G. B. Lamont, (2002), "Evolutionary algorithms for solving multiobjective problems", Kluwer Academic Publishers, New York, ISBN 03064-6762-3.

[8] C. A. Coello Coello and N. Cruz Cort'es, (2005), "Solving multiobjective optimization problems using an artificial immune system", Genetic Programming and Evolvable Machines, vol. 6 p 163-190, Springer Sinece + Business Media, Inc.

[9] J. F. Gieras, Z. J. Pieck, (1999), "Linear Synchronous Motors: Transportation and Automation Systems", CRC Press.

[10] Kaisa M. Miettinen, (2002), "Nonlinear MultiobjectiveOptimization", Kluwer Academic Publishers.

[11] L. Nunes de Castro and F. J. Von Zuben, (1999), "Artificial immune systems: Part I: Basic theory and applications", Technical Report TR-DCA 01/99, FEEC/UNICAMP, Brazil.

[12] L. Nunes de Castro and F. J. Von Zuben, (2001), "aiNet: An artificial immune network for data analysis", Data Mining: A Heuristic Approach, Idea Group Publishing, USA, pp. 231259.

[13] L. Nunes de Castro and F. J. Von Zuben, (2002), "Learning and optimization using the clonal selection principle", IEEE Transactions on Evolutionary Computation, vol. 6, no. 3, pp. 239-251.

[14] Marco Farina et al, (2001), "Cost-effective Evolutionary Strategies for Pareto Optimal Front Approximation in Multiobjective Shape Design Optimization of Electromagnetic Devices", Department of Electrical Engineering, UNIVERSITY OF PAVIA, Italy.

[15] R. Filomeno Coelho, (2004), "Multicriteria Optimization with Expert Rules for Mechanical Design", Dissertation, Université Libre De Bruxelles Faculté Des Sciences Appliquées.

[16] Rao S. S., (2009), "Engineering Optimization: Theory and Practice", $(4 r d$ ed). New York: Wiley.

[17] W. F. Abd El-Wahed, E. M. Zaki, A. El-Refaey, (2010), "Artificial Immune System based Neural Networks for Solving Multi-objective Programming Problems", Egyptian Information Journal Vol. (11) No. 2 pp. 59-65.

[18] W. F. Abd El-Wahed, E. M. Zaki, A. El-Refaey, (2010), "Reference Point Based Multi-Objective Optimization Using Hybrid Artificial Immune System", Universal Journal of Computer Science and Engineering Technology, Vol. (1) No. 1 pp: 24-30. 